\title{
More Information Needed on Probiotic Supplement Product Labels
}

\author{
Daniel Merenstein, MD ${ }^{1,2,3} \oplus^{0}$, John Guzzi, BS ${ }^{4}$, and Mary Ellen Sanders, PhD ${ }^{5}$ \\ 'Department of Family Medicine, Georgetown University, Washington, DC, USA; ${ }^{2}$ Research Programs Family Medicine, Georgetown University \\ Medical Center, Washington, DC, USA; ${ }^{3}$ Department of Human Science, School of Nursing and Health Studies, Georgetown University Medical \\ Center, Washington, DC, USA; ${ }^{4}$ Georgetown University Medical School, Washington, DC, USA; 5 Dairy \& Food Culture Technologies, Centennial, CO, \\ USA.
}

J Gen Intern Med 34(12):2735-7

DOI: $10.1007 / \mathrm{s} 11606-019-05077-5$

(c) Society of General Internal Medicine 2019

\section{INTRODUCTION}

Probiotics are live microorganisms that, when administered in adequate amounts, confer a health benefit on the host. ${ }^{1}$ Several specific probiotic strains are the subject of numerous robust studies, which comprise level 1 evidence. ${ }^{2,3}$ However, there have been some recent well-publicized trials in which probiotics did not fare better than placebo. ${ }^{4,5}$ We were interested in determining the extent to which retail probiotic dietary supplements were labeled in a manner that enabled us to determine if evidence supported product claims.

\section{METHODS}

The Georgetown University IRB granted exemption status for this study. Between October 2018 and March 2019, JG visited four large national probiotic retailers in the Washington, DC area. Probiotic foods were excluded. The following information was collected from the product packaging: recommended use, dosage/serving size, colony forming units (CFUs)/serving, storage information/instructions, genus/species/strain of probiotic, use by/expiration date. Product manufacturers were not contacted for additional supportive information. The information was reviewed independently by DM and MES to determine if the product claims were supported by clinical evidence. In order for products to be deemed supported by evidence, the product label had to disclose strain designation(s), the strain had to be present at an efficacious dose, and at least one controlled human study needed to be listed on PubMed.

\section{RESULTS}

Labels of 93 products from four stores were examined. Of the 93 products, 67 appeared to be unique (composed of distinct strains or combinations, regardless of $\mathrm{CFU} /$ dose or claims

Received May 1, 2019

Accepted May 6, 2019

Published online June 3, 2019 made) (Fig. 1). We judged 33 (35\%) of the 93 products to be supported by evidence for their stated claims. Of the 33 that were supported by the evidence, 18 provided potency guarantee through the end of shelf life (not at the time of manufacture). A higher number of strains, higher dose, or greater cost/ dose was not associated with evidence (Fig. 1). Indeed, a product with fewer strains was more likely to be supported by evidence (Fig. 2).

\section{DISCUSSION}

This analysis was based solely on label declarations, which indicated that a significant percentage $(65 \%)$ of retail probiotics cannot be linked to evidence of efficacy. It is possible that additional evidence supporting product claims exists, for example, on product websites or as unpublished data. In addition, it is possible that the product has evidence but without strain designations on the label, we could not confirm.

All products were labeled in compliance with FDA regulations. The FDA does not require strain designations or viable counts at the end of shelf life on a probiotic supplement label, although the California state legislature recently introduced legislation (CA bill AB1178) to require this on all probiotic dietary supplements marketed in California. Such requirements would increase transparency and improve traceability of evidence.

Specific claims on probiotics must be tied to specific strains. Because not all strains or doses can be expected to work for all indications, science-based organizations emphasize that the choice of probiotic should be based on evidence of health benefit. Too often, consumers think "more is better," motivating them to purchase based on the greatest number of strains or highest CFU. We found many high-dose, multiple-species, and more expensive products that lacked evidence.

This survey had several limitations. First, we did not evaluate the quality of the evidence. If one controlled trial showed benefit that matched at least one claim, we considered that sufficient. Greater scrutiny regarding study quality or clinical significance of results might lead others to conclude differently. Further, we did not do a systematic review of evidence; we relied on what was retrievable in PubMed. Second, we 


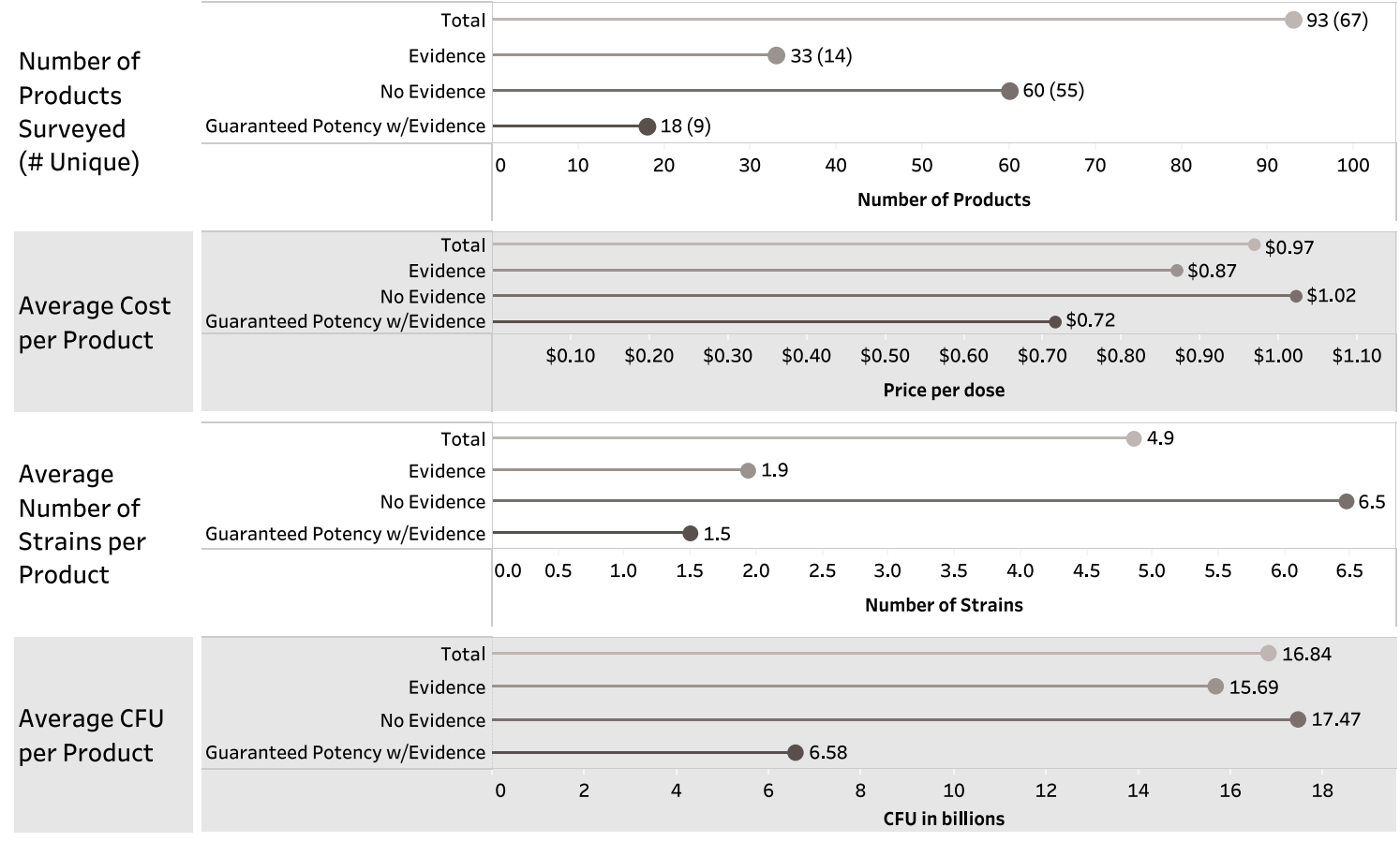

Figure 1 Information gathered from labels of samples of probiotics obtained at four retail outlets. Numbers in parentheses are the number of unique products for respective category. A product is listed as "without evidence" if it was not possible to tie the product as labeled with published literature; any product not listing strain designations was considered "without evidence." Determining if a product was unique was based on strain composition regardless of CFU/dose or claims. Products that did not stipulate strain designations were all considered unique, as the product composition at the strain level could not be determined from the label.

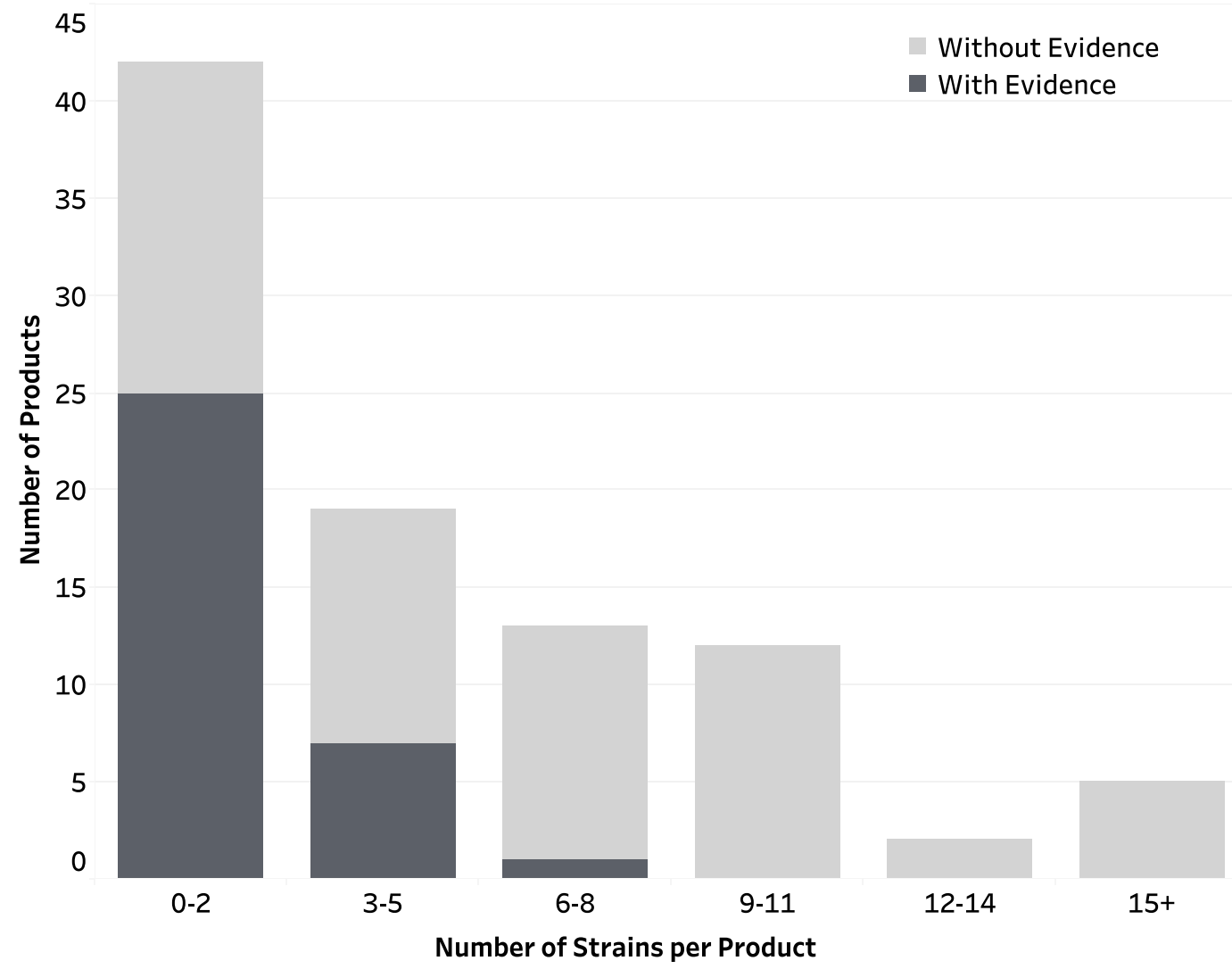

Figure 2 Number of strains per product with and without evidence. Surveyed products contained between 1 and 17 strains per product. Further, how each group was divided between products with and without evidence is shown. See legend in Figure 1 for explanations of evidence and unique. 
accepted any intestinal endpoint study as evidence to support a "digestive health" or similar claim.

A recent article questioned the safety and adequate regulation of probiotics in the USA. ${ }^{6}$ We did not assess safety of products, but all products contained commonly used genera and species, which have excellent safety records when used in the generally healthy population. Evidence of probiotic efficacy for several indications exists and future research is likely to expand the range of potential benefits. However, in order for healthcare providers and consumers to use probiotics in an evidence-based manner, labeling of many products should be improved.

Acknowledgments: The authors would like to gratefully acknowledge Dr. Alexandra Mannerings for designing the figures.

Corresponding Author: Daniel Merenstein, MD; Department of Human Science, School of Nursing and Health Studies Georgetown University Medical Center, Washington, DC, USA (e-mail:djm23@georgetown.edu).

\section{Compliance with Ethical Standards:}

Conflict of Interest: Dr. Sanders reports personal fees from California Dairy Research Foundation, personal fees from Nestle, personal fees from Danone Research, personal fees from The Dannon Company/ Danone North America, personal fees from Williams Mullen, personal fees from Winclove, personal fees from Pharmavite, personal fees from Yakult, personal fees from Dutch Mill, personal fees from Clorox, personal fees from Pfizer, personal fees from Medscape, personal fees from Probi, personal fees from Mead Johnson, personal fees from GSK, personal fees from Church \& Dwight, personal fees from General Mills, personal fees from Visalia Dairy Company, personal fees from International Scientific Association for Probiotics and Prebiotics, personal fees from Proctor \& Gamble, personal fees from Kelly Dry \& Warren LLP, non-financial support from United States Pharmacopeia, non-financial support from International Life Sciences Institute, personal fees from Kellogg, personal fees from Trouw Pet Nutrition, personal fees from JHeimbach LLC, and personal fees from Kerry, outside the submitted work.

Dr. Merenstein has received personal fees from Bayer, Pharmavite, Reckitt Benckiser, and Debevoise \& Plimpton.

John Guzzi has nothing to disclose.

\section{REFERENCES}

1. Hill C, Guarner F, Reid G, et al. Expert consensus document. The International Scientific Association for Probiotics and Prebiotics consensus statement on the scope and appropriate use of the term probiotic. Nat Rev Gastroenterol Hepatol. 2014;11(8):506-514.

2. Goldenberg JZ, Mertz D, Johnston BC. Probiotics to Prevent Clostridium difficile Infection in Patients Receiving Antibiotics. JAMA. 2018;320(5):499-500.

3. Sung V, D'Amico F, Cabana MD, et al. Lactobacillus reuteri to Treat Infant Colic: A Meta-analysis. Pediatrics. 2018;141(1).

4. Freedman SB, Williamson-Urquhart S, Farion KJ, et al. Multicenter Trial of a Combination Probiotic for Children with Gastroenteritis. N Engl J Med. 2018;379(21):2015-2026.

5. Schnadower D, Tarr PI, Casper TC, et al. Lactobacillus rhamnosus GG versus Placebo for Acute Gastroenteritis in Children. N Engl J Med. 2018;379(21):2002-2014.

6. Cohen PA. Probiotic Safety-No Guarantees. JAMA Intern Med. 2018;178(12):1577-1578.

Publisher's Note Springer Nature remains neutral with regard to jurisdictional claims in published maps and institutional affiliations. 